Journal of the Magnetics Society of Japan Vol. 15 Supplement, No. S2 (1991)

(C) 1991 by The Magnetics Society of Japan

\title{
A NEW SUPPORTING MECHANISM FOR NON-FLYING SINGLE-POLE HEAD
}

\author{
Shinya FUJIMATSU, Isao WATANABE, Hiroaki MURAOKA \\ Kazuhiro OUCHI and Yoshihisa NAKAMURA
}

\author{
Research Institute of Electrical Communication, Tohoku University \\ 2-1-1, Katahira, Aoba-ku, Sendai 980, JAPAN
}

\begin{abstract}
We have studied two types of head supporting mechanisms for a non-flying single-pole-type head (SPT head). One is a self-weight type, in which the smooth up-and-down motion of a head is achieved using a micro linear bearing. The other is a balanced type that achieves the access to both sides of the disks and make the disk access stable under acceleration over $1 \mathrm{G}$. Both new supporting mechanisms show a good off-track and a track access characteristics. After the seeking of $7 \mathrm{~mm}$ distance on a disk, a reproduced voltage fluctuation with the new type mechanism is less than $2 \%$ that corresponds to the off-track distance of $2 \mu \mathrm{m}$, whereas, that with the conventional type mechanism is $5 \%-10 \%$ that corresponds to the off-track over $10 \mu \mathrm{m}$. A head installed in this head supporting mechanisms follows the disk up to 3600 r.p.m.. This result agrees well with the calculated results of the head motion using the equation of motion.
\end{abstract}

\section{INTRODUCTION}

A head-to-medium spacing must be as narrow as possible to realize high areal bit density perpendicular magnetic recording $[1,2]$. We have examined the headto-medium interface in contact recording with the nonflying SPT head on a hard disk $[3,4]$. In this system, the head is in contact with the medium at a load force of the weight of an up-down moving-element, as shown in Fig.1. As compared with a flexible disk system, the flat envelopes of reproduced signals are easily obtained, which means that the stable spacing about $30 \mathrm{~nm}$ are obtained. The spacing could be reduced and be constant for a relative velocity up to $10 \mathrm{~m} / \mathrm{s}$ [5].

However, when an excessive clearance exists between the moving-element and the holder, the serious offtracking and the head vibration have occurred after and during track seeking. Furthermore, these head supporting mechanisms accessed only the upper side surface of disks. The head access to the lower side surface of disks becomes possible if gimbals are introduced [6]. Such a support mechanism needs big spaces above a disk. An SPT head will not follow a disk surface under over $1 \mathrm{G}$ disturbance to the disk drives. To improve these problems, we propose two types of the supporting mechanisms. One type is designed to make movement of the moving-element smooth. Another is developed to make an SPT head to easily access both surfaces of a disk with light load force $0.5 \mathrm{gf}$.

\section{STRUCTURE OF HEAD SUPPORTING MECHANISMS}

\section{Self-weight type mechanism}

Fig.2(a) shows an advanced self-weight type mechanism. In this mechanism, a micro linear bearing was installed between the moving-element and the holder, and an SPT head chip was put on the tip of the movingelement. The element moves in the up and down directions very smoothly without any play. The head loading force depends only on the mass of the movingelement including a head itself. Table 1 shows the list of the weight and the shape for various moving elements. A guide-pin is set to prevent the rotation of the movingelement.

\section{Balanced type mechanism}

Fig.2(b) shows a balanced-type mechanism. The head arm is supported to keep its balance, and a miniature bearing is used as a pivot. An SPT head was put on the end of the head arm. The head arm turns smoothly and easily on the pivot. A spring is used to apply a loading force on an SPT head to improve a following property to a disk. By adjusting this spring, the SPT head is contact with an upper and a lower sides of a disk. 


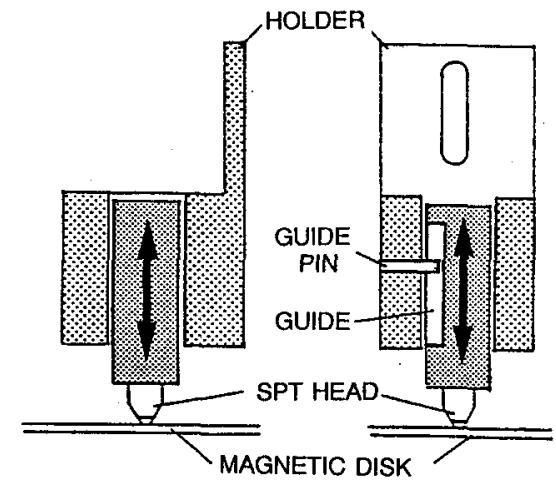

Table 1 A moving-element weight

\begin{tabular}{|c|c|c|}
\hline material & shape $(3 \phi)$ & weight(mg) \\
\hline \hline carbon fiber & rod & 240 \\
\hline aluminum & tube & 240 \\
\hline aluminum & rod & 320 \\
\hline brass & tube & 550 \\
\hline brass & rod & 880 \\
\hline iron & rod & 820 \\
\hline
\end{tabular}

Fig.1 A cross-sectional view of the conventional self-weight type supporting mechanism.

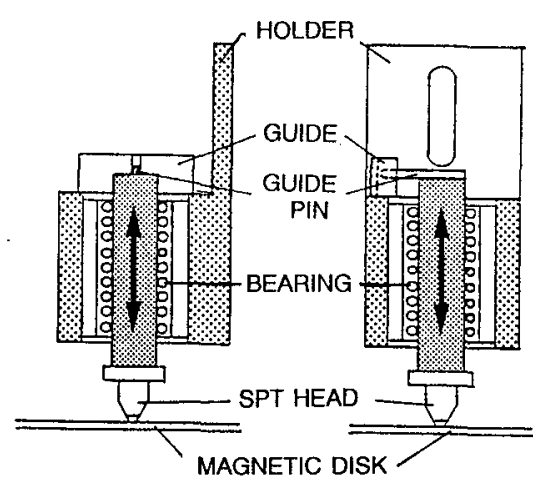

(a) The self-weight type (cross sectional view)

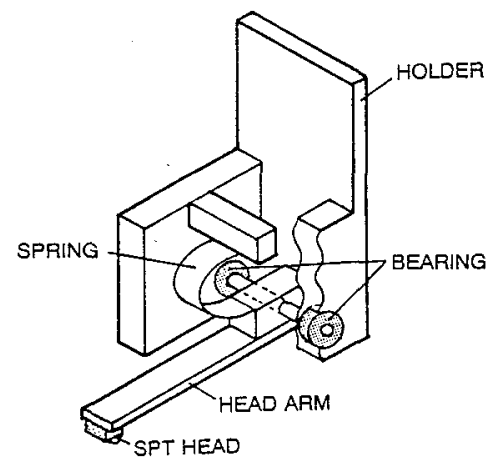

(b) The balanced type

Fig.2 The structures of the new type supporting mechanisms.

\section{HEAD MOTION ANALYSIS BY EQUATION OF MOTION}

\section{Model 1}

We modelled this supporting mechanism as the massspring system as shown in Fig.3, where $m$ is a mass of an SPT head, $k$ is a spring constant, $x$ is position of the center of gravity of the SPT head, and $y$ is position of the disk surface. The force applied to a head is an inertial resistance $-m \ddot{x}$, a spring force $-k x$, a gravity $-m g$, a load force $-F_{l}$ and an external force from a disk $F$. Thus, the equation of motion is given by

$$
-m \ddot{x}-k x-m g-F_{l}+F=0 .
$$

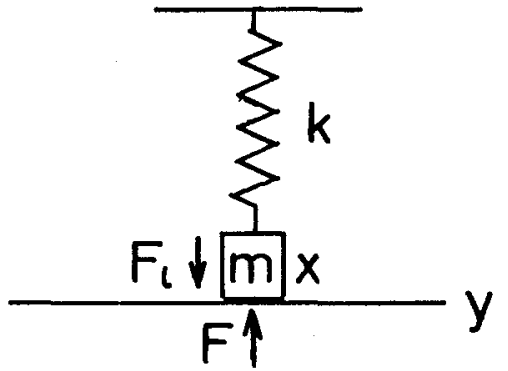

Fig.3 A model of supporting mechanism.

$$
\begin{aligned}
& k: \text { spring constant } \\
& m: \text { mass of head } \\
& x: \text { position of center of gravity } \\
& y: \text { position of disk surface } \\
& F_{l}: \text { load force } \\
& F: \text { external force from disk }
\end{aligned}
$$


To solve the equation (1), a disk surface motion is assumed as sinusoid:

$$
y=a * \sin (2 \pi f t),
$$

where $a$ is an amplitude of motion, $f$ is a frequency of disk motion and $t$ is a time.

To calculate the locus of the head and the external force to apply to the head from the disk, the equation (1) is solved by a numerical method. By transforming the equation (1) and the acceleration $x$ expressed a second differential of a head position $x$, an external force $F$ is represented by

$$
F_{i}=m \frac{x_{i}-2 x_{i-1}+x_{i-2}}{d t^{2}}+k x_{i}+m g+F_{i},
$$

where subscript $i$ denotes a value at time $i$.

When $F \geq 0$, the head is pressed against the disk. Namely, the head is in contact with a disk. Then the head position $x_{i}$ is equal to the disk position $y_{i}$.

$$
x_{i}=y_{i}
$$

On the other hand, when the external force $F$ becomes as $F<0$, this means that the head is pulled apart from the disk. Namely, the head is separated from the disk. As the external force $F$ never takes the negative value, in such case $F$ must be $F=0$.

A head position $x_{i}$ is expressed by

$$
x_{i}=\frac{1}{\frac{m}{d t^{2}+k}}\left(\frac{m}{d t^{2}}\left(2 x_{i-1}-x_{i-2}\right)-m g-F_{l}\right) .
$$

\section{Calculation of head motion}

In a supporting mechanism without a spring, it is assumed that a spring constant, an SPT head mass and a load force are $0 \mathrm{~N} / \mathrm{m}, 0.55 \mathrm{~g}$ and $0 \mathrm{gf}$, respectively. Using these values, a head position and an external force from the disk are calculated from equations (2) to (5), whereas amplitude of a disk surface motion is assumed to be $20 \mu \mathrm{m}$. When the acceleration over $1 \mathrm{G}$ acts to a head, a head jumps over a disk and crashes to a disk. In Fig.4, a disk motion, an SPT head locus and an external force from the disk are shown by a solid, a dash and a dot-dash lines, respectively. This means that the head cannot be in stable contact with the disk when an exciting force over $1 \mathrm{G}$ acceleration that acts to a disk drive.

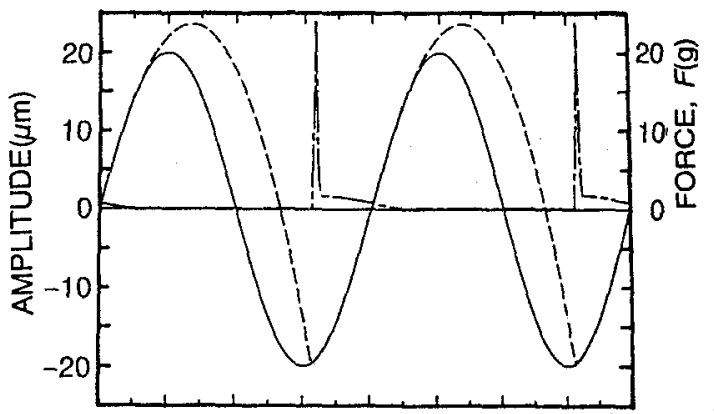

Fig.4 Head motion in a supporting mechanism without a spring, calculated at disturbance over $1 \mathrm{G}$. A disk motion, a head locus and an external force from the disk are shown by a solid, a dash and a dot-dash lines, respectively.

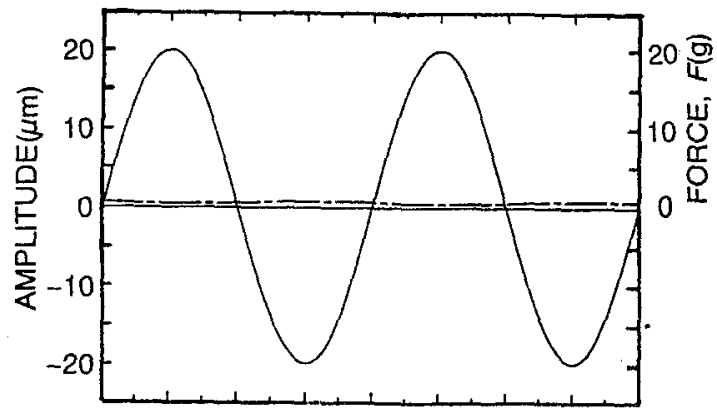

Fig.5 Head motion in a supporting mechanism without a spring, calculated at disturbance under $1 \mathrm{G}$. A disk motion, a head locus and an external force from the disk are shown by a solid, a dash and a dot-dash lines, respectively.

But the maximum acceleration is estimate to be about $0.3 \mathrm{G}$, when a disk rotates at 3600 r.p.m.. The head completely follows the disk fluctuation as shown in Fig.5, unless the any other external force is applied. Thus a solid and a dash lines in Fig.5 are overlapped. In this case, it is proved that a head is in contact with a disk.

On the other hand, in the supporting mechanism with a spring, it is assumed that a spring constant, a head mass, a load force and a disk surface motion amplitude are $20 \mathrm{~N} / \mathrm{m}, 0.5 \mathrm{~g}, 0.5 \mathrm{gf}$ and $20 \mu \mathrm{m}$, respectively. Using these values, a head position and an external force from the disk are calculated from equation (2) to (5). In this case, an SPT head keeps be contact with the disk even for over $1 \mathrm{G}$ acceleration. Thus, using the balanced type mechanism with the light load spring, the stable head contact with a disk is possible. 


\section{RESULTS AND DISCUSSION}

\section{Self-weight type mechanism}

In order to evaluate the supporting mechanism, a head off-track test was performed. First a signal of $20 \mathrm{kFRPI}$ was recorded on a track. Then the reproduced output was measured with shifting the head across the recorded track. In Fig.6, the measured results for the new and the old mechanism are shown by a solid and a dash lines, respectively. In the old type mechanism, the holder position for the maximum output is shifted by about $5 \mu \mathrm{m}$ from the center of the recorded track. This result means that there is a play of $5 \mu \mathrm{m}$ between the head and the holder which will cause fatal miss tracking in narrow track recording. On the other hand, in the new mechanism, a head can precisely trace the recorded tracks.

Since the track seeking performance is an important factor for drives as well as an off-track characteristics, it was examined in the following manner. The $20 \mathrm{kFRPI}$ signal was recorded on a track, and then the head was moved away by the travelling distance of $1 \mathrm{~mm}$ to $7 \mathrm{~mm}$. After the head holder was returned to the originally recorded position, the reproduced voltage was measured. In Fig.7, the reproduced voltages, normalized by that just after recording are plotted with open circles and triangles for the new and the old mechanisms, respectively. In the old mechanism, the reproduced voltage was reduced by $5-10 \%$ of the original reproduced voltage because of the low accuracy of the track seeking. On the other hand, in the new mechanism, the reproduced voltages were almost same as high as the original value within the accuracy of $1 \%$, even after seeking of the distance of $7 \mathrm{~mm}$. This voltage reduction corresponds to the off-track less than $2 \mu \mathrm{m}$. Hence, it is clear that precise head positioning was realized by introducing this new mechanism.

Fig.8 shows the disk surface following property of the moving-element in the new supporting mechanism. The disk surface fluctuation and the displacement of the moving-element in the up and down directions were measured separately by an optical sensor. The disk rotating speed was 3600 r.p.m.. In this figure, we can estimate the amplitude of the disk surface fluctuation to be about $20 \mu \mathrm{m}$. The movement of the moving-element coincided with the disk surface fluctuation even at 3600 r.p.m.. Thus, we confirmed that the moving-element realizes stable contact with the rotating disk surface.

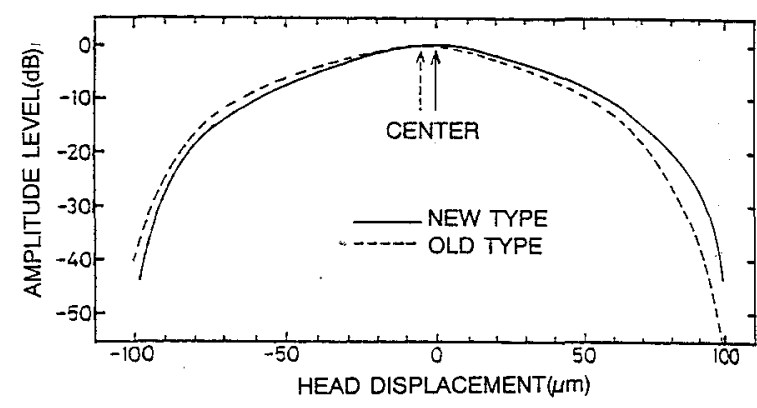

Fig.6 An off-track characteristics.

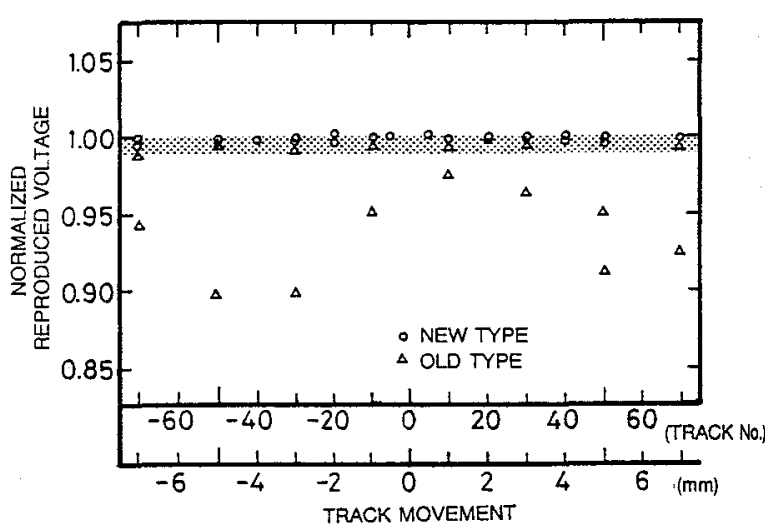

Fig.7 A track seeking characteristics.

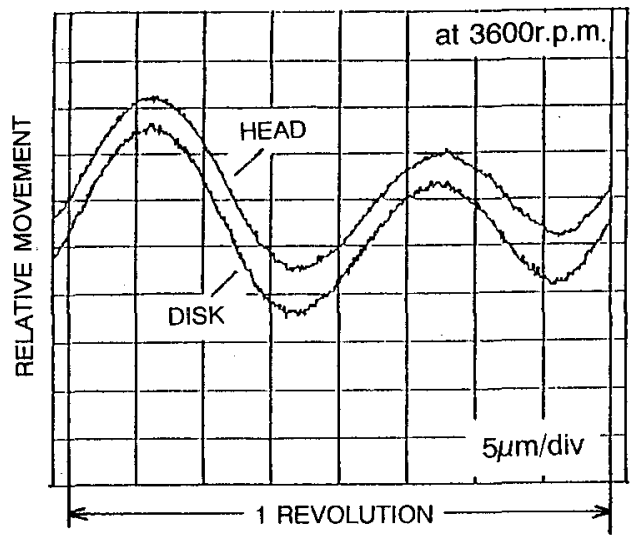

Fig.8 A bead and a disk surface movement over a revolution of disk measured by by an optical sensor. 
Furthermore, the reproduced voltage was measured for various head/medium relative velocities. A $5 \mathrm{kFRPI}$ signal was first recorded on a track at head/medium velocity of $2 \mathrm{~m} / \mathrm{s}$, and then head/medium velocity was increased up to $11 \mathrm{~m} / \mathrm{s}$ in reproduction. In Fig.9, the reproduced voltages, normalized by each head/medium relative velocity and the reproduced voltage value at $2 \mathrm{~m} / \mathrm{s}$, are plotted by open circles. The normalized reproduced voltage did not change although the velocity was increased up to $11 \mathrm{~m} / \mathrm{s}$. We can conclude that the head-to-medium spacing is constant and the moving-element completely follows the disk surface fluctuation in the new self-weight type mechanism.

\section{Balanced type mechanism}

Fig.10 shows the bit density response curves measured by a balanced type mechanism. The measurement was made using both upper and lower side heads. The voltages reproduced by the upper and the lower side heads and normalized by number of tums, track width and head/medium velocity are plotted by an open and a solid squares, respectively, in Fig.10. The same bit density response is obtained by both sides of heads.

The reproduced voltage was measured with changing head/medium relative velocity. A digital NRZI signal at $5 \mathrm{kFRPI}$ was first recorded on a track at a head/medium relative velocity of $2 \mathrm{~m} / \mathrm{s}$. Then the reproduced voltage was observed with the velocity up to $11 \mathrm{~m} / \mathrm{s}$. In Fig.11, the reproduced voltages, normalized by each head/medium relative velocity and a reproduced voltage value at $2 \mathrm{~m} / \mathrm{s}$, were plotted by open and solid squares, respectively. For the upper and the lower side access, the reproduced voltage did not change in this velocity range. Thus, in this velocity range, the head-to-medium spacing keeps the same value of about $30 \mathrm{~nm}$ as that measured by a light interference method in a static state. The balanced type mechanism was realized a stable contact recording and reproducing on both sides of a media in wide range of velocity.

In order to check the play of the head-arm, the head off-track test was carried out by the same experimental procedure as that used for the self-weight type mechanism. The results is shown in Fig.12. A head position for a maximum output precisely coincides with the center of the originally recorded track.

Track seeking performance was also examined by the same method as that used for the self-weight type

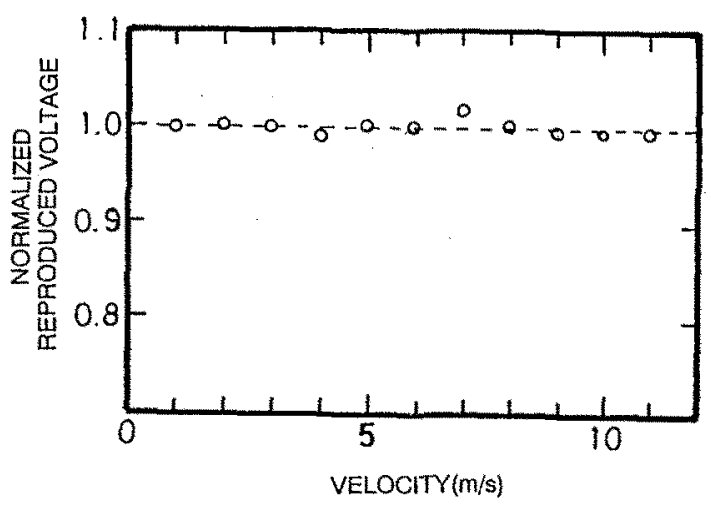

Fig.9 An output voltage normalized by velocity versus head-to-medium relative velocity.

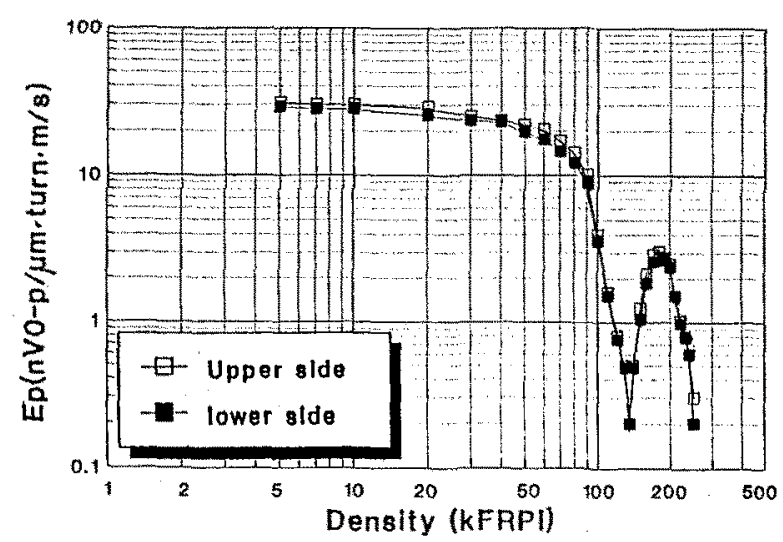

Fig.10 Bit density response curves for upper and lower side access head at the recording magnetomotive-force of $1 \mathrm{AT}_{\mathrm{P}-\mathrm{P}}$.

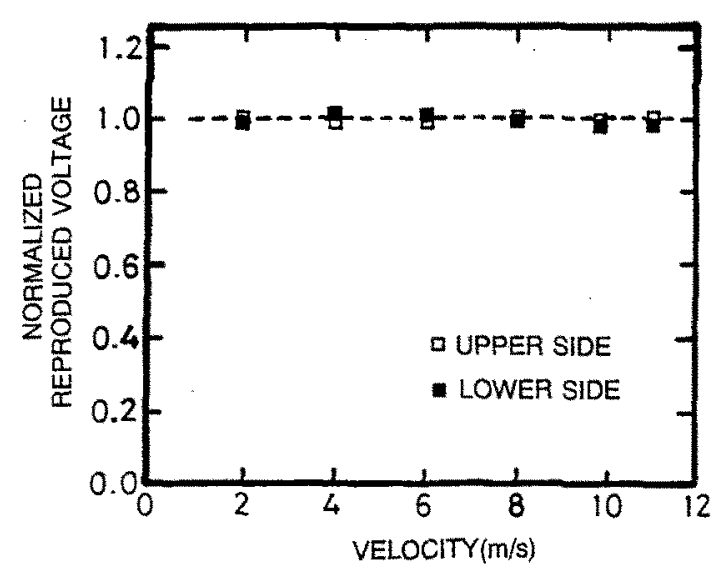

Fig.11 An output voltage normalized by velocity versus head-to-medium relative velocity. 
mechanism. The reproduced voltage, normalized by that just after recording, are plotted in Fig.13. The reproduced voltage did not change within an accuracy of $1 \%$, even after seeking with the distance of $7 \mathrm{~mm}$. This experimental result proves that a very smooth and a precise head positioning is realized by the balanced type mechanism.

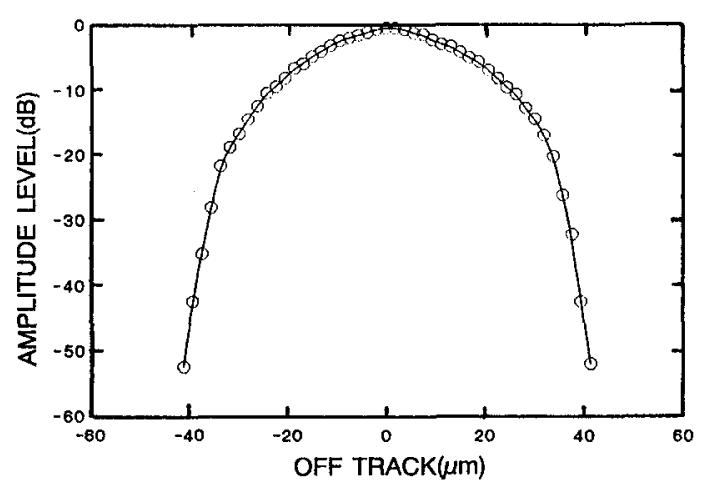

Fig.12 An off-track characteristics.

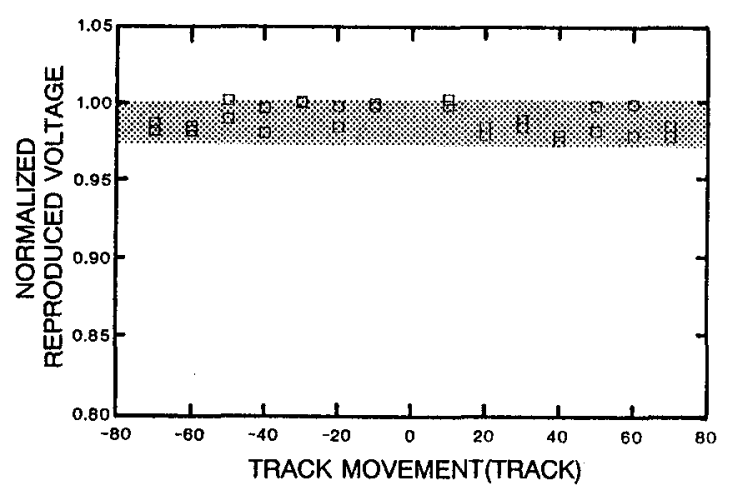

Fig.13 A track seeking characteristics.

\section{CONCLUSIONS}

The new supporting mechanisms for non-flying SPT head was proposed: one is the self-weight type which realizes a smooth up-down movement using bearings, another is the balanced type which realizes stable disk access for both sides of a medium. These new head supporting mechanisms demonstrated precise head positioning and stable head seeking. These mechanisms are useful for a contact narrow track recording.

For the contact recording type hard disk system, the importance of the smooth movement and the firm mechanical supporting of heads and the light mass of head have been emphasized. When these conditions are satisfied, the heads can follow the disk surface in contact without serious wear problem. For the light heads, a small loading force applied by a spring, is sufficient to ensure the tolerance against the acceleration disturbance.

\section{ACKNOWLEDGEMENTS}

We would like to thank Dr. S. Yamamoto for their invariable discussions. We also acknowledge Mr. K. Takano and M. Otagiri for making single-pole heads.

\section{REFERENCES}

[1] S.Yamamoto, Y.Nakamura, S.Iwasaki, "Flexible magnetic disk image file system using perpendicular magnetic recording", IEEE Trans. Magn., Vol. MAG-23, pp.2070-2072, September 1987.

[2] Y.Nakamura, K.Ouchi, S.Yamamoto, I.Watanabe, "Recording characteristics of perpendicular magnetic hard disk measured by non-flying single-pole Head", IEEE Trans. Magn., Vol.26, pp.2436-2438, September 1990.

[3] H.Fukuoka, Y.Nakamura, "Evaluation of rigid perpendicular magnetic disks using the non-flying type magnetic head", IEICE Technical Report MR89-4, pp.1-7, 1989.

[4] Y.Nakamura, K.Ouchi, I.Watanabe, "Evaluation of perpendicular magnetic rigid disk by non-flying singlepole head", Journal of the Magnetics society of Japan, Vol.14, pp.135-138, 1990.

[5] S.Yamamoto, I.Watanabe, K.Ouchi, Y.Nakamura "Perpendicular magnetic rigid disk system using nonflying type single-pole head", IEICE Technical Report MR90-14, pp.31-38, 1990.

[6] S.Yamamoto, I.Watanabe, K.Ouchi, Y.Nakamura "Head-disk interface in perpendicular magnetic hard disk system using non-flying single-pole head", 1990 Autumn national convention record, IEICE, SC-5-1, pp.5-268 5-269, 1990. 